\title{
P71. Adoptive transfer of TCR gene-transduced lymphocytes targeting MAGE-A4 for refractory esophageal cancer
}

\author{
H Shiku ${ }^{1 *}$, H Ikeda ${ }^{1}$, Y Miyahara', M Ishihara ${ }^{2}$, N Katayama² ${ }^{2}$ D Tomura ${ }^{3}$, I Nukaya ${ }^{3}$, J Mineno ${ }^{3}$, K Takesako $^{3}$, \\ S Kageyama ${ }^{1}$
}

From 1st Immunotherapy of Cancer Conference (ITOC1)

Munich, Germany. 12-14 March 2014

\section{Background}

Engineering the antigen receptor gene in patients' lymphocytes is one promising strategy to create antigen-specific lymphocytes without senescent phenotypes. The strategy provides an opportunity to extend the application of adoptive $\mathrm{T}$ cell therapy for cancer patients. However, this concept has not been tested in the epithelial cancer patients.

\section{Materials and methods}

MAGE-A4-specific TCR $\alpha$ and $\beta$ chains were cloned from a human $\mathrm{T}$ cell clone that recognises MAGEA4 143-151 peptide in a HLA-A*24:02 restricted manner. This $\mathrm{T}$ cell clone did not show any cross reactivity to the peptides with homology to the MAGE-A4 $4_{143-151}$ epitope. A retroviral vector that encodes these TCR chains without any artificial modification was constructed; the lymphocytes transduced with the retroviral vector killed the MAGE-A4 expressing tumor in vitro and inhibited the tumour growth in the NOG immunodeficient mice.

A phase I clinical trial of TCR gene therapy targeting MAGE-A4 was performed to treat refractory esophageal cancer patients without lympho-depleting pre-conditioning. The trial was designed as a cell-dose escalation consisting of three cohorts, $2 \times 10^{8}, 1 \times 10^{9}$ and $5 \times 10^{9}$ cells/patient. Vaccines with the cognate peptide were also given following adoptive transfer of lymphocytes on day 14 and day 28 . lymphocytes were detected in their peripheral blood in a dose-dependent manner during the first 14 days. In 4 patients, the infused cells have been persisting more than 5 months after the transfer. The transferred lymphocytes that were harvested from the patients more than 50 days after the transfer were found to sustain the reactivity to the antigen-expressing tumour cells. Three patients showed SD or long tumour free status.

\section{Conclusions}

This approach may extend the availability of adoptive $\mathrm{T}$ cell therapy for epithelial cancer patients by providing tumour-reactive and long surviving lymphocytes.

\section{Authors' details}

'Mie University Graduate School of Medicine, Cancer Vaccine and ImmunoGene Therapy, Tsu Mie, Japan. ${ }^{2}$ Mie University Graduate School of Medicine, Hematology and Oncology, Tsu Mie, Japan. ${ }^{3}$ Takara Bio Inc., Center for Cell and Gene Therapy, Shiga Mie, Japan.

Published: 12 March 2014

\section{doi:10.1186/2051-1426-2-S2-P45}

Cite this article as: Shiku et al:: P71. Adoptive transfer of TCR gene-transduced lymphocytes targeting MAGE-A4 for refractory esophageal cancer. Journal for ImmunoTherapy of Cancer 2014 2(Suppl 2): P45.

\section{Results}

The treatment was tolerable with no adverse events associated with transferred cells or any viral toxicity. In all ten patients of the 3 cell-doses, the transferred

Mie University Graduate School of Medicine, Cancer Vaccine and ImmunoGene Therapy, Tsu Mie, Japan

Full list of author information is available at the end of the article 